Mathématiques et sciences humaines
Mathematics and social sciences

191 | Automne 2010

Variabilité et inégalités

\title{
Introduction au numéro spécial : «Variabilité et inégalités »
}

\section{OpenEdition}

Journals

Édition électronique

URL : http://journals.openedition.org/msh/11850

DOI : $10.4000 / \mathrm{msh} .11850$

ISSN : 1950-6821

Éditeur

Centre d'analyse et de mathématique sociales de l'EHESS

\section{Édition imprimée}

Date de publication : 1 septembre 2010

Pagination : 5

ISSN : 0987-6936

Référence électronique

"Introduction au numéro spécial : «Variabilité et inégalités » 》, Mathématiques et sciences humaines [En ligne], 191 | Automne 2010, mis en ligne le 15 février 2011, consulté le 23 juillet 2020. URL : http:// journals.openedition.org/msh/11850 


\section{VARIABILITÉ ET INÉGALITÉS}

En novembre 2009 s'est tenu à la Sorbonne un colloque dont le sujet était les mesures de la dispersion et de la concentration. Il se voulait ainsi complémentaire de celui que quelques collègues (B.-P. Lécuyer, B. Matalon...) avaient organisé sur la « moyenne » en $1989^{1}$. Une moyenne ne commence à avoir du sens que si on lui adjoint une mesure de la dispersion des observations autour d'elles. C'est cela que ce colloque a voulu pallier.

Le présent numéro est constitué de certaines des interventions à ce colloque. Celles des interventions qui ont un caractère historique sont publiées dans le J.E.H.P.S. (http://www.emis.de/journals/JEHPS/index-2.html). Nous espérons pouvoir prochainement publier les autres.

M.B.

\section{Programme du colloque \\ DISPERSION (CONCENTRATION), VARIABILITÉ, INÉGALITÉS ${ }^{2}$}

19 novembre - 21 novembre 2009

Jeudi 19 novembre - Méthodes statistiques, leur histoire, leurs enjeux

M. BARBUT (CAMS - EHESS) : De quoi parlons-nous ? Problèmes, méthodes, outils

J. BAsulto et J. Busto (Dept. d'économie appliquée, Université de Séville) : Corrado Gini (18841965) et son indice de concentration (1908-1914)

M. Armatte (Centre Alexandre Koyré - EHESS et Université Paris-Dauphine) : Statut de la dispersion. De l'erreur à la variabilité individuelle

Vendredi 20 novembre - Perception et représentations

B. VALADE (GEPECS - Université Paris-Descartes) : La contribution de Paul Leroy-Beaulieu à l'étude de la répartition des richesses

M. FoRsÉ (Centre Maurice Halbwachs - ENS - EHESS - CNRS) : La perception des inégalités économiques dans huit pays développés

É. BRIAN (Centre Maurice Halbwachs - ENS - EHESS - CNRS): Probabilités subjectives et dispersion dans les phénomènes financiers

\section{Géographie, Psychologie, Biologie}

Mme D. PUMAIN (UMR Géographie-Cités - Université de Paris I) : La dynamique des inégalités en géographie et les changements d'échelle

P. VRIGNAUD (Université de Paris Ouest Nanterre-La Défense): Différences inter et intraindividuelles en psychologie

J. GAYON (Institut d'histoire et de philosophie des sciences et des techniques - Université de Paris I) : Incidence des paramètres de dispersion en biologie de l'évolution.

B. DERRIDA (Université P. et M. Curie et Laboratoire de physique statistique - ENS) : Le rôle des fluctuations en physique statistique

Samedi 21 novembre - Démographie, Sociologie, Économie

L.A. VALLET (CREST - INSEE) : Quelques aspects statistiques en sociologie, 1950-2010

A. TRANNOY (GREQAM - EHESS, Marseille) : Mesures d'inégalité et dominance sociale

D. COURGEAU (INED) : La dispersion des mesures démographiques : vue historique

\footnotetext{
${ }^{1}$ «Moyenne, milieu et centre. Histoires des usages », colloque parisien organisé en 1989 à l'initiative de la Société française pour l'histoire des sciences de l'Homme.

${ }^{2}$ Organisé par le séminaire d'histoire du calcul des probabilités et de la statistique (EHESS), le Groupe d'étude pour l'Europe de la culture et de la solidarité (GEPECS - Université Paris Descartes), le Centre d'analyse et de mathématique sociales (CAMS - EHESS) et l'École doctorale de sciences humaines et sociales (Université Paris Descartes).
} 